\title{
A REATIVIDADE DO ESTADO EXCITADO TRIPLETE DE 1,3-INDANODIONA EM BENZENO
}

Frances R. dos Santos, Mônica T. da Silva e José Carlos Netto-Ferreira*

Departamento de Química, Universidade Federal Rural do Rio de Janeiro, Antiga Rod. Rio-São Paulo km 47, 23851-970

Seropédica - RJ, Brasil

Nanci C. de Lucas

Departamento de Química Orgânica, Universidade Federal do Rio de Janeiro, Cidade Universitária, Ilha do Fundão,

21949-970 Rio de Janeiro - RJ, Brasil

Recebido em 17/5/06; aceito em 24/8/06; publicado na web em 26/3/07

TRIPLET EXCITED STATE REACTIVITY OF 1,3-INDANDIONE IN BENZENE SOLUTION. Rate constants for the quenching of 1,3-indandione (1) triplet by olefins and by hydrogen and electron donors were obtained employing the laser flash photolysis technique in benzene solution. These rate constants ranged from $2.5 \times 10^{5} \mathrm{Lmol}^{-1} \mathrm{~s}^{-1}$ (for 2-propanol) to $5.9 \times 10^{9} \mathrm{Lmol}^{-1} \mathrm{~s}^{-1}$ (for DABCO). From the quenching rate constants by 1,3-cyclohexadiene, trans- and cis-stilbene a value between 49.3 and $52.4 \mathrm{kcal} / \mathrm{mol}$ was estimated for the energy of the triplet state of 1,3-indandione. The $n \pi^{*}$ character of this triplet state was evidenced by the quenching rate constants obtained when typical hydrogen donors were employed as quenchers. For 2-phenyl-1,3-indandione (2, R=phenyl) a fast Norrish type I reaction is operating which prevents the determination of kinetic and spectroscopic data of its triplet state.

Keywords: 1,3-indandiones; laser flash photolysis; triplet excited state.

\section{INTRODUÇÃO}

1,3-indanodiona (1) e seus derivados contendo grupos arila na posição 2 , isto é $\mathbf{2}$, são de grande interesse tecnológico face às suas propriedades antioxidantes, como reguladores de reações de polimerização e como estabilizadores de polímeros ${ }^{1,2}$. Estes processos envolvem a participação dos radicais livres centrados no carbono formados a partir da quebra homolítica da ligação C2-H, ou seja, os radicais 1,3-indanodion-2-ila (3). Esta quebra homolítica pode ser feita tanto através da fotólise das 1,3-indanodionas quanto pela termólise dos seus dímeros 2,2'-indanodionila ${ }^{3,4}$.

O envolvimento destes radicais é também o responsável pelas diversas propriedades farmacológicas apresentadas por 1,3indanodionas, tais como ação sobre o sistema nervoso central ${ }^{5}$, inibição da biossíntese de prostaglandinas ${ }^{6}$ e da atividade de $\beta$ glucuronidase $^{7}$, fosforilação oxidativa de mitocôndrias ${ }^{8}$, modificadores de amino ácidos e peptídeos ${ }^{9}$ ou ação anti-coagulante ${ }^{10}$.

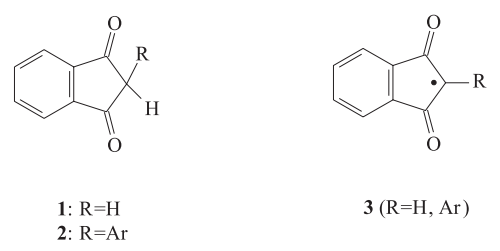

Apesar do grande interesse acerca das propriedades do radical 1,3-indanodion-2-ila (3) pouco se sabe acerca da reatividade do precursor (1) no estado excitado triplete.

Este trabalho teve como objetivo estudar a reatividade do estado excitado triplete de 1,3-indanodiona (1) em benzeno frente a supressores que podem se comportar como doadores de hidrogênio, tais como 1,4-cicloexadieno, 2-propanol, tolueno, fenol, doadores de elétron, isto é, 1,4-diazabiciclo[2.2.2]octano (DABCO) e olefinas.

\footnotetext{
*e-mail: jcnetto@ufrrj.br
}

\section{PARTE EXPERIMENTAL}

\section{Materiais}

Os solventes benzeno e acetonitrila (Aldrich Chemical Co., grau espectroscópico), bem como os reagentes tolueno, 2-propanol e fenol (Merck) foram usados tais como recebidos. 1,3-indanodiona, 1,4-diazabiciclo[2.2.2] octano (DABCO), 1,1-difenileteno, transestilbeno e cis-estilbeno foram adquiridos da Aldrich e também usados como recebidos. 1,4-cicloexadieno e 1,3-cicloexadieno (Aldrich) foram destilados bulbo-a-bulbo, a pressão reduzida, antes da sua utilização.

\section{Técnicas gerais}

Os espectros na região do UV-visível foram obtidos em espectrofotômetro Varian Cary 4E. Os experimentos de fotólise por pulso de laser foram realizados em instrumento analítico da Edinburgh modelo LP900. As amostras foram colocadas em cela de quartzo de $10 \times 10 \mathrm{~mm}$, tendo sido deaeradas com $\mathrm{N}_{2}$ livre de oxigênio por 20 min e irradiadas com laser Surelite Nd/YAG, usando-se o terceiro harmônico $(\lambda=355 \mathrm{~nm})$ com pulso de $5 \mathrm{~ns}$ e $40 \mathrm{~mJ}$. A concentração das amostras foi escolhida de modo a fornecer uma absorção no comprimento de onda de excitação $(355 \mathrm{~nm})$ com densidade óptica de aproximadamente 0,5 .

Os experimentos de supressão foram realizados a partir de soluções estoque do supressor, de modo que somente fosse necessário adicionar volumes da ordem de $\mu \mathrm{L}$ à célula para se obter concentrações adequadas do supressor. Os experimentos foram realizados na sua maioria em benzeno, a não ser que o emprego de qualquer outro solvente esteja assinalado no texto.

\section{RESULTADOS E DISCUSSÃO}

A fotólise por pulso de laser de uma solução de 1,3-indanodiona (1) em benzeno levou à formação de um transiente com máximos 
de absorção a 320 e 700 nm (Figura 1). Este transiente decai com cinética de primeira ordem apresentando tempo de vida de $5 \mu$ s (Figura 1, inserção) e foi suprimido por 1,3-cicloexadieno $\left(\mathrm{E}_{\mathrm{T}}=\right.$ $\left.52,4 \mathrm{kcal} \mathrm{mol}^{-1}\right)^{11}$ com uma constante de velocidade de $3,0 \times 10^{8} \mathrm{~L}$ $\mathrm{mol}^{-1} \mathrm{~s}^{-1}$, o que indica que se trata do estado excitado triplete de $\mathbf{1}$. É interessante assinalar que não foi observado o decaimento de segunda ordem comum a cetonas aromáticas e resultante do processo de aniquilação triplete-triplete, o que pode ser devido à baixa concentração inicial de 1,3-indanodiona.

O decaimento deste triplete em presença deste e dos outros supressores empregados neste trabalho segue uma cinética de pseudoprimeira ordem, com a constante de velocidade medida experimentalmente $\left(\mathrm{k}_{\mathrm{obs}}\right)$ estando relacionada à constante de velocidade de supressão segundo a equação de Stern-Volmer ${ }^{12}$ (Equação 1).

$\mathrm{k}_{\mathrm{obs}}=\mathrm{k}_{0}+\mathrm{k}_{\mathrm{q}}[\mathrm{Q}]$

onde $\mathrm{k}_{0}$ é a constante de velocidade de decaimento do estado excitado triplete na ausência de supressor, $\mathrm{k}_{\mathrm{q}}$ é a constante de velocidade de decaimento do estado excitado triplete na presença do supressor e [Q] é a concentração do mesmo.

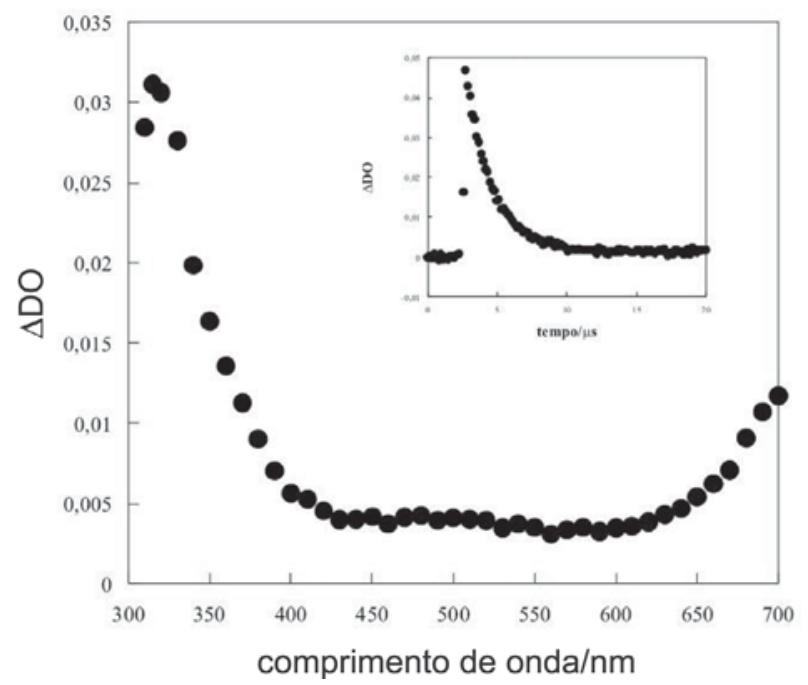

Figura 1. Espectro de absorção para o transiente gerado a partir da excitação de uma solução 0,01 mol $L^{-1}$ de 1,3-indanodiona (1) em benzeno em célula de fluxo. Inserção: decaimento do triplete monitorado a $320 \mathrm{~nm}$

O baixo valor encontrado para a constante de velocidade de supressão do triplete de $\mathbf{1}$ por 1,3-cicloexadieno, menor que a constante de velocidade no limite difusional neste solvente, indica que este triplete deve ter energia menor que a do supressor, uma vez que processos exergônicos de transferência de energia são controlados pela difusão das espécies no meio, ou seja, o estado excitado triplete do doador e o estado fundamental do supressor. Para benzeno, a $25^{\circ} \mathrm{C}$, o valor da constante de velocidade no limite difusional, segundo a teoria de Smoluchowski ${ }^{11}$, é de $\sim 10^{10} \mathrm{~L} \mathrm{~mol}^{-1} \mathrm{~s}^{-1}$.

O caráter triplete do transiente obtido na fotólise de 1,3indanodiona foi confirmado pelos estudos de supressão empregando-se o $\beta$-caroteno $\left(\mathrm{E}_{\mathrm{T}}=19,0 \mathrm{kcal} \mathrm{mol}^{-1}\right)^{13}$. Este polieno tem cruzamento entre sistemas iguais a zero ${ }^{14} \mathrm{e}$, portanto, o seu estado triplete só pode ser formado através de um processo de transferência de energia a partir de um doador adequado. Uma vez que $\beta$ caroteno tem um espectro de absorção triplete-triplete com uma absorção máxima a $520 \mathrm{~nm}$ (Figura 2), o processo de supressão do triplete de 1 por esta espécie pode ser facilmente monitorado se- guindo-se tanto o decaimento mono-exponencial do triplete de $\mathbf{1}$, a 315 ou $700 \mathrm{~nm}$, quanto o crescimento mono-exponencial do triplete de $\beta$-caroteno a $520 \mathrm{~nm}$ (Figura 2, inserção), como uma função da concentração do supressor. Em ambos os casos observou-se uma constante de velocidade de supressão controlada por difusão, o que nos permite confirmar a natureza triplete do transiente obtido após a excitação por laser de 1,3-indanodiona em benzeno.

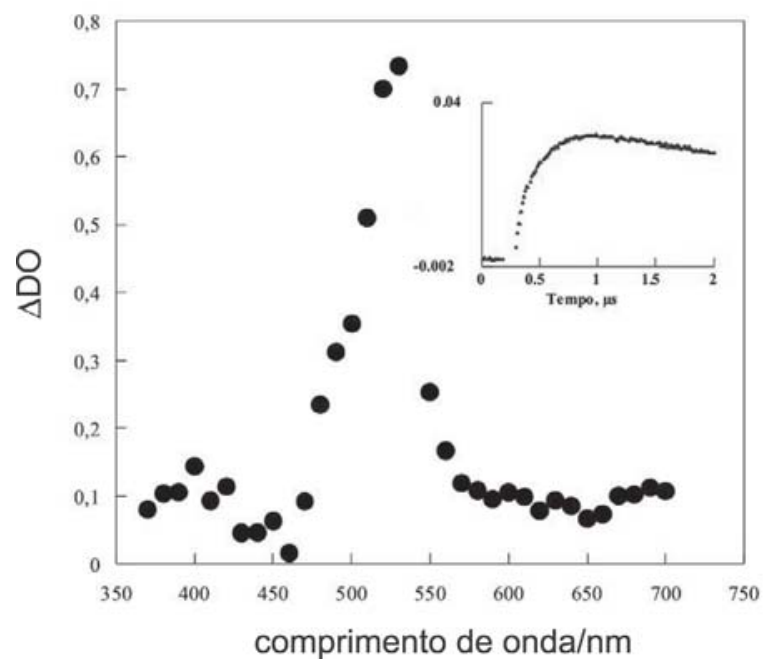

Figura 2. Espectro de absorção triplete-triplete para $\beta$-caroteno gerado por transferência de energia de 1,3-indanodiona. Inserção: crescimento do sinal relativo ao triplete de $\beta$-caroteno monitorado a $520 \mathrm{~nm}$

Uma constante de velocidade de supressão abaixo do valor da constante de velocidade difusional também foi obtida quando se empregou cis-estilbeno $\left(\mathrm{E}_{\mathrm{T}}=54,3 \mathrm{kcal} \mathrm{mol}^{-1}\right)^{11}$ como supressor (Tabela 1). Entretanto, para trans-estilbeno $\left(\mathrm{E}_{\mathrm{T}}=49,3 \mathrm{kcal} \mathrm{mol}^{-1}\right)^{11} \mathrm{o}$ valor medido para a constante de velocidade de supressão aproxima-se do limite difusional. Este conjunto de dados cinéticos acerca do processo de transferência de energia nos permite concluir que a energia do estado excitado triplete para 1,3-indanodiona se situa entre 49,3 e $52,4 \mathrm{kcal} \mathrm{mol}^{-1}$.

Tabela 1. Constantes de velocidade $\left(\mathrm{k}_{\mathrm{q}}\right)$ para a supressão do estado excitado triplete de 1,3-indanodiona (1) por diversos supressores, em benzeno

\begin{tabular}{lc}
\hline Supressor & $\mathrm{k}_{\mathrm{q}}\left(\mathrm{L} \mathrm{mol}^{-1} \mathrm{~s}^{-1}\right)^{\mathrm{a}}$ \\
\hline 1,3-cicloexadieno & $3,0 \times 10^{8}$ \\
$\beta$-caroteno & $6,0 \times 10^{9}$ \\
trans-estilbeno & $4,6 \times 10^{9}$ \\
cis-estilbeno & $2,3 \times 10^{8}$ \\
2-propanol & $2,0 \times 10^{5}$ \\
1,4-cicloexadieno & $9,3 \times 10^{6}$ \\
tolueno & $5,7 \times 10^{6}$ \\
fenol & $3,3 \times 10^{8}$ \\
DABCO & $5,5 \times 10^{9}$ \\
1,1-difenileteno & $1,0 \times 10^{7}$ \\
\hline
\end{tabular}

a Erro estimado: $\pm 10 \%$

A característica $n \pi *$ do estado excitado triplete de 1,3-indanodiona pode ser evidenciada pelos valores das constantes de velocidade de supressão $\left(\mathrm{k}_{\mathrm{q}}\right.$ ) obtidos quando se empregam, como supressor, doadores típicos de hidrogênio, tais como 1,4-cicloexadieno, 2-propanol e tolueno. Para estes supressores foram obtidas constantes de velocida- 
de de supressão de $9,3 \times 10^{6}, 2,0 \times 10^{5}$ e $5,7 \times 10^{6} \mathrm{~L} \mathrm{~mol}^{-1} \mathrm{~s}^{-1}$, respectivamente. A Figura 3 mostra um exemplo representativo do gráfico de Stern-Volmer, empregando a Equação 1, para a supressão de $\mathbf{1}$ por 1,4-cicloexadieno. Incluso nesta figura está também o gráfico de supressão desta mesma dicetona por DABCO.

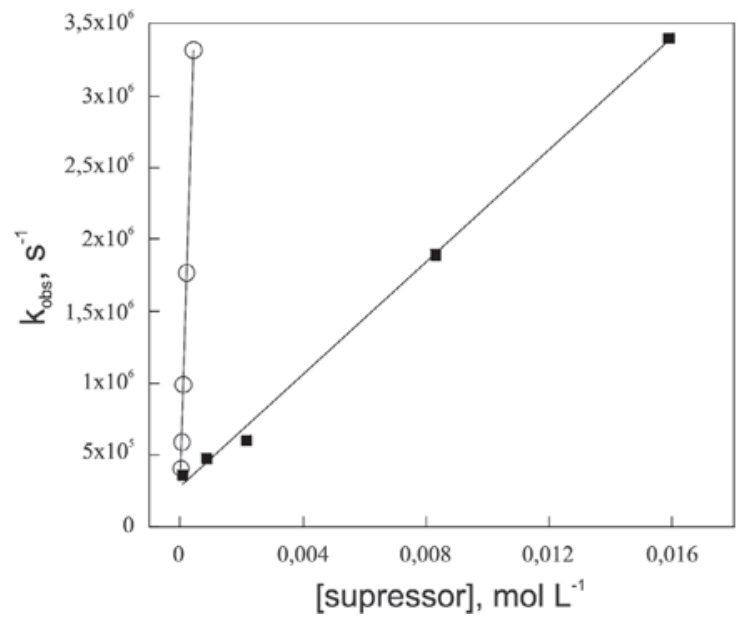

Figura 3. Gráficos de Stern-Volmer para a supressão de 1,3-indanodiona em benzeno por 1,4-cicloexadieno (-) e DABCO (O)

Nos casos da supressão pelos doadores de hidrogênio citados anteriormente, um mecanismo de transferência de hidrogênio, semelhante àquele observado quando se emprega radical alcoxila como modelo para carbonilas excitadas $n \pi * 15$, deve estar operando, não podendo se descartar, no caso da abstração do hidrogênio benzílico do tolueno, o envolvimento de um complexo no estado excitado, com certo grau de transferência de carga, entre 1,3indanodiona e tolueno ${ }^{16}$.

A fotólise de 1,3-indanodiona (1) em presença de 1,4cicloexadieno resulta na formação de um novo transiente, com máximo de absorção abaixo de $320 \mathrm{~nm}$ (Figura 4) e que deve ser associado ao radical cetila (correspondente ao 3 no Esquema 1), já que para dienos não conjugados, como no caso de 1,4-cicloexadieno, o processo primário envolvido na fotólise de cetonas corresponde a uma reação de abstração de hidrogênio alílico. Considerando que o

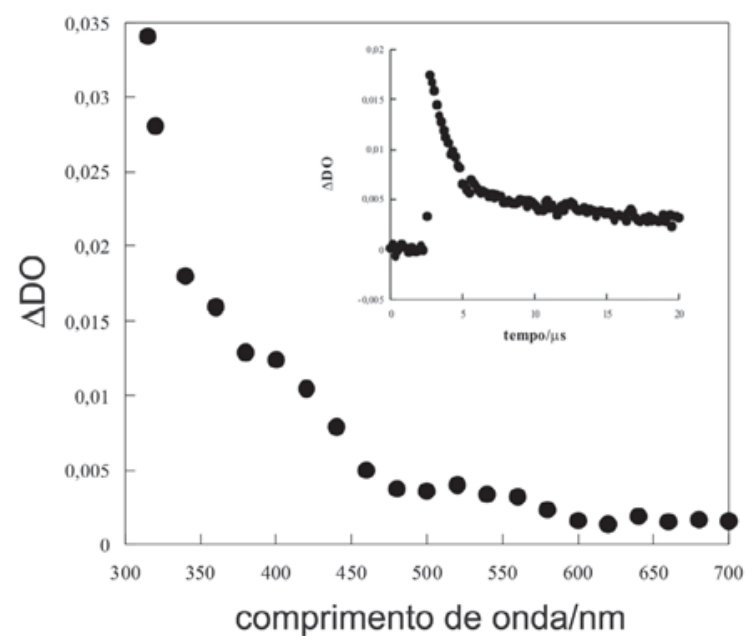

Figura 4. Espectro de absorção para o transiente gerado a partir da excitação de uma solução 0,01 mol $L^{-1}$ de 1,3-indanodiona em benzeno, em presença de 1,4-cicloexadieno. Inserção: decaimento correspondente ao triplete de 1 e ao seu radical cetila, monitorado a $320 \mathrm{~nm}$

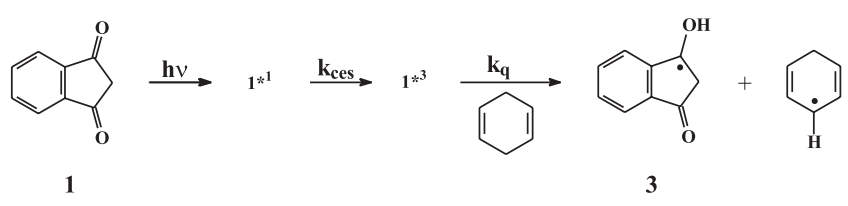

Esquema 1

radical cetila e o estado excitado triplete de 1 têm espectros transientes sobrepostos, a cinética associada a este processo de supressão tornase razoavelmente complexa. O decaimento inserido na Figura 3, e obtido em presença de uma concentração de 1,4-cicloexadieno de cerca de $2 \mathrm{mmol} \mathrm{L}^{-1}$, mostra claramente que ele é constituído por dois componentes: um decaimento rápido, correspondente ao estado excitado triplete que está sendo suprimido, e um outro, mais lento, associado ao radical cetila, que usualmente tem tempo de vida mais longo que o do triplete correspondente. Em concentrações de supressor nas quais o triplete está quase que completamente suprimido ( $>90 \%$ ), pode-se observar somente o decaimento longo correspondente ao radical cetila.

A constante de velocidade de supressão por fenol $\left(3,3 \times 10^{8}\right.$ $\mathrm{L} \mathrm{mol}^{-1} \mathrm{~s}^{-1}$ ) é de 2 a 3 ordens de grandeza superior à observada para os supressores citados (Tabela 1), o que indica que o mecanismo de supressão neste caso é distinto daquele proposto anteriormente para processos primários ocorrendo por transferência de hidrogênio. A abstração de hidrogênio fenólico por estados excitados triplete de carbonilas normalmente é mais rápida que aquela observada para toluenos, uma vez que fenóis apresentam tanto uma menor energia de dissociação da ligação $\mathrm{O}-\mathrm{H}$, comparada à $\mathrm{C}-\mathrm{H}$, quanto um menor potencial de oxidação que toluenos. Assim, o mecanismo da reação de abstração de hidrogênio de fenóis deve ocorrer através da formação inicial de um exciplexo triplete, estabilizado por ligação de hidrogênio, seguido por uma transferência seqüencial de elétron e de próton ${ }^{17-25}$.

1,4-diazabiciclo[2.2.2] octano (DABCO) suprime de maneira eficiente $\left(k_{q}=5,5 \times 10^{9} \mathrm{~L} \mathrm{~mol}^{-1} \mathrm{~s}^{-1}\right.$, Tabela 1, Figura 4) o estado excitado triplete de 1,3-indanodiona (1) através um processo de transferência de elétron, levando à formação do ânion radical da dicetona, isto é 4, e do cátion radical derivado da amina (Esquema 2). Uma vez que DABCO não tem átomos de hidrogênio facilmente transferíveis devido a efeitos estereoeletrônicos desfavoráveis, não é possível a formação de radicais cetila quando a fotólise de $\mathbf{1}$ é realizada em presença deste supressor.

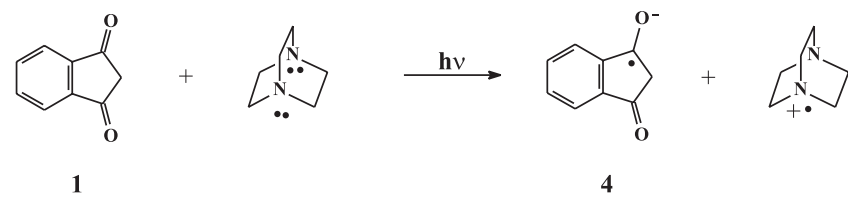

Esquema 2

A adição à solução de 1,3-indanodiona em benzeno de 1,1difenileteno também levou à supressão do estado excitado triplete de $1\left(\mathrm{k}_{\mathrm{q}}=1,0 \times 10^{7} \mathrm{~L} \mathrm{~mol}^{-1} \mathrm{~s}^{-1}\right.$, Tabela 1). Entretanto, é importante assinalar que neste caso a constante de velocidade de supressão é de 1 a 2 ordens de grandeza menor que as observadas para cis- e trans-estilbeno, para os quais o processo de supressão envolve transferência de energia intermolecular. No caso de 1,1-difenileteno, portanto, a supressão provavelmente envolva uma reação de fotocicloadição $[2 \pi+2 \pi]$ (reação de Patterno-Büchi) ${ }^{26}$, através da formação de um birradical 1,4 (Esquema 3). 


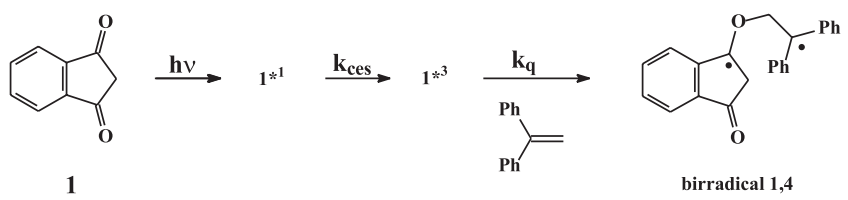

Esquema 3

Estudos por fotólise por pulso de laser para 2-fenil-1,3indanodiona $(2, \mathrm{R}=$ fenila) em acetonitrila, usando-se sistema de fluxo, não resultaram na formação de nenhum transiente. Isto é compatível com resultados anteriores da literatura que indicam que esta dicetona sofre reação de clivagem $\alpha$ (Tipo I de Norrish) de modo extremamente eficiente, resultando na formação do ftalídeo $5^{27}$ (Esquema 4). Quando o experimento foi realizado em célula estática, observou-se a formação de uma espécie cujo espectro de absorção na região do UV-visível é idêntico ao observado após a irradiação de $\mathbf{2}$ ( $\mathrm{R}=$ fenila) por 24 h e que foi previamente atribuído ao ftalídeo $\mathbf{5}^{27}$.
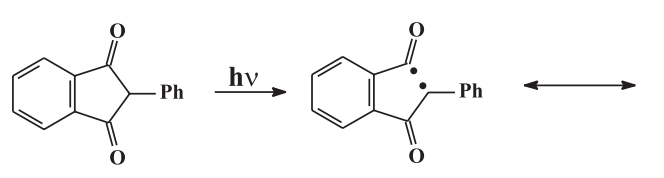

1
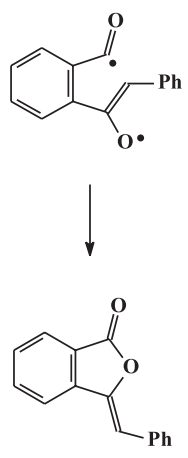

5
Esquema 4

Em conclusão, foi demonstrado empregando-se a técnica de fotólise por pulso de laser de nanossegundo que o estado excitado triplete de 1,3-indanodiona (1) tem energia triplete entre 49,3 e 52,4 $\mathrm{kcal} \mathrm{mol}^{-1}$, sendo suprimido por supressores que sejam doadores de hidrogênio ou de elétron e também por olefinas, com constantes de velocidade de supressão variando de $2,0 \times 10^{5} \mathrm{~L} \mathrm{~mol}^{-1} \mathrm{~s}^{-1}$ (2-propanol) a $5,5 \times 10^{9} \mathrm{~L} \mathrm{~mol}^{-1} \mathrm{~s}^{-1}$ (DABCO). Por outro lado, dados espectroscópicos e cinéticos para 2-fenil-1,3-indanodiona (2) não puderam ser obtidos, uma vez que esta dicetona sofre reação de clivagem $\alpha$ de maneira eficiente, fornecendo o ftalídeo correspondente.

\section{AGRADECIMENTOS}

Ao suporte financeiro fornecido pelo Conselho Nacional de Desenvolvimento Científico e Tecnológico (CNPq) e Fundação Carlos Chagas Filho de Amparo à Pesquisa do Estado do Rio de Janeiro (FAPERJ). M. T. da Silva agradece à Fundação Coordenação para o Aperfeiçoamento do Pessoal de Nível Superior (CAPES) e J. C. Netto-Ferreira ao CNPq pelas bolsas concedidas.

\section{REFERÊNCIAS}

1. Moiseev, V. V.; Poluetkov, I. T.; Usp. Khim. 1973, 42, 471.

2. Nishiyama, T.; Shiotsu, S.; Tsujita, H.; Polym. Degrad. Stab. 2002, 76, 435.

3. Zechner, J.; Getoff, N.; Timcheva, I.; Fratev, F.; Minchev, S.; Z. Naturforsch., A: Phys. Sci. 1983, 38, 1337.

4. Timcheva, I.; Nikolov, P.; Zechner, J.; Getoff, N.; Minchev, S.; Z. Naturforsch., A: Phys. Sci. 1987, 42, 490.

5. Cavallini, G.; Milla, E.; Grumelli, E.; Rabean, F.; Farmaco 1955, 10, 710.

6. van der Berg, G.; Bultsma, T.; Nauta, W. Th.; Biochem. Pharmacol. 1975, $24,1115$.

7. van der Berg, G.; Winter, M. L.; De Boer, W. A.; Nauta, W. Th.; Biochem. Pharmacol. 1976, 25, 1397.

8. van der Berg, G.; Volters, D.; Nauta, W. Th.; Biochem. Pharmacol. 1975, $24,1397$.

9. Aleksiev, B.; Mincev, St.; J. Prakt. Chem. 1974, 316, 140.

10. Zechner, J.; Grabnes, G. I.; Köhler, G.; Getoff, N.; Timcheva, I.; Fratev, F.; Minchev, S.; J. Photochem. 1983, 23, 61.

11. Murov, S. L.; Carmichael, I.; Hug, G. L. Handbook of Photochemistry, $2^{\text {nd }}$ ed.; Marcel Dekker, Inc.: New York, 1993.

12. Stern, O.; Volmer, M.; Physik. Z. 1919, 20, 183.

13. Bensansson, R.; Land, E. J.; Mavdinas, B.; Photochem. Photobiol. 1976, 23, 189.

14. Kumar, C. V.; Das, P. K.; J. Am. Chem. Soc. 1983, 105, 5143.

15. Griller, D.; Howard, J. A.; Marriott, P. R.; Scaiano, J. C.; J. Am. Chem. Soc. 1981, 103, 619.

16. Wagner, P. J.; Truman, R. J.; Puchalski, A. E.; Wake, R.; J. Am. Chem. Soc. 1986, 108, 7727.

17. Turro, N. J.; Engel, R.; J. Am. Chem. Soc. 1969, 91, 7113.

18. Das, P. K.; Encinas, M. V.; Scaiano, J. C.; J. Am. Chem. Soc. 1981, 103, 4154.

19. Leigh, W. J.; Lathioor, E. C.; St-Pierre, M. J.; J. Am. Chem. Soc. 1996, 118, 12339.

20. Biczok, L.; Berces, T.; Linschitz, H.; J. Am. Chem. Soc. 1997, 119, 11071.

21. Jovanovic, S. V.; Morris, D. G.; Pliva, C. N.; Scaiano, J. C.; J. Photochem. Photobiol., A 1997, 107, 153.

22. de Lucas, N. C.; Netto-Ferreira, J. C.; J. Photochem. Photobiol., A 1998, 116, 203.

23. Serra, A. C. S.; de Lucas, N. C.; Netto-Ferreira, J. C.; J. Braz. Chem. Soc. 2004, 15, 481 .

24. Silva, M. T.; Netto-Ferreira, J. C.; J. Photochem. Photobiol., A 2004, 162, 225.

25. Lathioor, E. C.; Leigh, W. J.; Photochem. Photobiol. 2006, 82, 291

26. Gilbert, A.; Baggot, J.; Essentials of Molecular Photochemistry, Blackwell Scientific Publications: Londres, 1991.

27. Rigaudy, J.; Derible, P.; Bull. Soc. Chim. Fr. 1965, 3047; Rigaudy, J.; Derible, P.; Bull. Soc. Chim. Fr. 1965, 3055; Rigaudy, J.; Derible, P.; Bull. Soc. Chim. Fr. 1965, 3061. 